University of Wollongong

Research Online

Faculty of Engineering and Information

Faculty of Engineering and Information

Sciences - Papers: Part A

Sciences

$1-1-2014$

Constitutive analysis for hot deformation behaviour of novel bimetal consisting of pearlitic steel and low carbon steel

Xingjian Gao

University of Wollongong, xg306@uowmail.edu.au

Zhengyi Jiang

University of Wollongong, jiang@uow.edu.au

Dongbin Wei

University of Technology Sydney, dwei@uow.edu.au

Huijun Li

University of Wollongong, huijun@uow.edu.au

Sihai Jiao

Baosteel, Baosteel Research Institute

See next page for additional authors

Follow this and additional works at: https://ro.uow.edu.au/eispapers

Part of the Engineering Commons, and the Science and Technology Studies Commons

Research Online is the open access institutional repository for the University of Wollongong. For further information contact the UOW Library: research-pubs@uow.edu.au 


\title{
Constitutive analysis for hot deformation behaviour of novel bimetal consisting of pearlitic steel and low carbon steel
}

\begin{abstract}
To understand the high temperature flow behaviour of a novel pearlitic steel (PS) and low carbon steel (LCS) bimetal, hot compression tests in a wide range of temperature and strain rate were conducted on a Gleeble 3500 thermo mechanical simulator, and the constitutive model was developed based on the experimental data. The measured true stress-strain curves exhibited three types of variation patterns, which are (i) a plateau type, (ii) single peak type and (iii) multi peaks type. These patterns well displayed the effects of the deformation temperature, strain rate and plastic strain on the flow behaviour of the bimetal. By incorporating the Zener-Hollomon parameter and material parameter functions of $\alpha(\varepsilon), n(\varepsilon)$, $Q(\varepsilon)$ and $A(\varepsilon)$ into Arrhenius-type constitutive equation, the flow stress values predicted by the proposed model show a good agreement with experimental results by the evidence of reproducing true stress-strain curves accurately, high value of correlation coefficient $(R=0.9873)$ and low value of average absolute relative error (AARE $=4.81 \%$ ). The proposed constitutive equation can be used to realise numerical simulation and determine processing parameters during hot-working of the PS/LCS bimetal.
\end{abstract}

\section{Keywords}

low, steel, pearlitic, consisting, bimetal, novel, carbon, behaviour, constitutive, deformation, hot, analysis

\section{Disciplines}

Engineering | Science and Technology Studies

\section{Publication Details}

Gao, X. J., Jiang, Z. Y., Wei, D. B., Li, H. J., Jiao, S. H., Xu, J., Zhang, X. M., Han, J. T. \& Chen, D. F. (2014). Constitutive analysis for hot deformation behaviour of novel bimetal consisting of pearlitic steel and low carbon steel. Materials Science and Engineering A: Structural Materials: Properties, Microstructure and Processing, 595 1-9.

\section{Authors}

Xingjian Gao, Zhengyi Jiang, Dongbin Wei, Huijun Li, Sihai Jiao, Jianzhong Xu, Xiaoming Zhang, Jingtao Han, and Dengfu Chen 


\title{
Constitutive analysis for hot deformation behaviour of novel bimetal consisting of pearlitic steel and low carbon steel
}

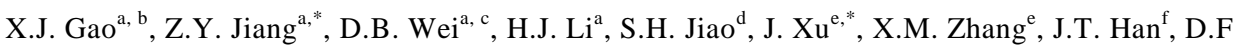

Chen ${ }^{\mathrm{b}}$

${ }^{a}$ School of Mechanical, Materials and Mechatronic Engineering, University of Wollongong, Wollongong NSW 2522,

Australia

${ }^{\mathrm{b}}$ College of Materials Science and Engineering, Chongqing University, Chongqing 400044, China

' School of Electrical, Mechanical and Mechatronic Systems, University of Technology, Sydney NSW 2007, Australia

${ }^{\mathrm{d}}$ Baosteel Research Institute, Baoshan Iron \& Steel Co Ltd, Shanghai 200941, China

e State Key Laboratory of Rolling and Automation, Northeastern University, Shenyang 110004, China

${ }^{\mathrm{f}}$ School of Materials Science and Engineering, University of Science \& Technology Beijing, Beijing 100083, China

*Corresponding authors: Z.Y. Jiang; Tel: 6124221 4545; Fax: 612 42215474; Email: jiang@uow.edu.au

J. Xu; Tel: 8624 83687220; Fax: 8624 23906472; Email: xujz@ral.neu.edu.au

\begin{abstract}
To understand the high temperature flow behaviour of a novel pearlitic steel (PS) and low carbon steel (LCS) bimetal, hot compression tests in a wide range of temperature and strain rate were conducted on a Gleeble 3500 thermo mechanical simulator, and the constitutive model was developed based on the experimental data. The measured true stress-strain curves exhibited three types of variation patterns, which are (i) a plateau type, (ii) single peak type and (iii) multi peaks type. These patterns well displayed the effects of the deformation temperature, strain rate and plastic strain on the flow behaviour of the bimetal. By incorporating the Zener-Hollomon parameter and material parameter functions of $\alpha(\varepsilon), n(\varepsilon), Q(\varepsilon)$ and $A(\varepsilon)$ into Arrhenius-type constitutive equation, the flow stress values predicted by the proposed model show a good agreement with experimental results by the evidence of reproducing true stress-strain curves accurately, high value of correlation coefficient $(\mathrm{R}=0.9873)$ and low value of average absolute relative error $(A A R E=4.81 \%)$. The proposed constitutive equation can be used to
\end{abstract}


realise numerical simulation and determine processing parameters during hot-working of the PS/LCS bimetal.

Keywords: Constitutive equation; Pearlitic steel; Bimetal; Prediction of flow stress

\section{Introduction}

Hypo eutectoid steels with high volume fraction of pearlitic structure and a small quantity of ferrite have been widely used in applications where reasonable strength and wear resistance are demanded. However, poor ductility and toughness as well as inferior weld ability are always keeping a lid on their further utilisations [1-4]. Recently, a novel bimetal consisting of hypo eutectoid pearlitic steel (PS) and low carbon steel (LCS) was developed to overcome those shortcomings by absorbing the advantages (i.e. excellent ductility and superior weld ability) of the latter component [5]. In order to improve the mechanical properties of the bimetal, various thermo mechanical treatments such as hot rolling and forging processes are generally proceeded on the bimetallic billet. However, the coexistence of harder PS and softer LCS in a layered structure of the bimetal makes the hot deformation behaviour different from that of the constituent components. Bonding of dissimilar steels into a single bimetal inevitably creates the interface and results in macro-heterogeneity of plastic deformation due to the discrepant hot-working responses. Therefore, it is of vital importance to study the high temperature flow behaviour of this novel bimetal.

Increasing recognition has been given to the constitutive equation as an effective method for predicting the material's flow behaviour based on a limited number of experimental data [6-20]. This mathematical representation is successfully used in the finite element code to simulate the deformation of the material under specified loading conditions. Over the past years, some efforts have been done on the development of constitutive equations for metal matrix composites (MMCs) by means of torsion or isothermal compression tests in a wide range of temperature and strain rate [15-17]. Spigarelli et al. [15] studied the hot-working response of an $\mathrm{Al}-6061 / 20 \% \mathrm{Al}_{2} \mathrm{O}_{3}$ composite using torsion tests and presented a consistent constitutive equation for both creep and hot deformation. Shao et al. [16] proposed the modified Arrhenius-type constitutive equation for 20 vol. \% SiC $/ 2024 \mathrm{Al}$ composite by analysing the 
experimental data derived from hot compression tests. Wang et al. [17] performed the hot compression tests on $\mathrm{Al}_{18} \mathrm{~B}_{4} \mathrm{O}_{33 \mathrm{w}} / \mathrm{AZ91D}$ composite and a modified Arrhenius-type constitutive equation was obtained. Despite an increase of attention is invested into the MMCs for clarifying their hot deformation behaviours, few investigations have been carried out to model the constitutive flow pattern of layer-structural bimetal, which is one of the metal matrix laminated composites.

In this paper, isothermal compression tests of a novel PS/LCS bimetal have been conducted at different temperatures and strain rates to characterise the flow behaviour during hot deformation. On the basis of the experimental results, a phenomenological approach [21] was applied to formulate the Arrhenius-type constitutive equation in which the flow stress was expressed by the hyperbolic laws and the strain compensation [22] was considered. The reliability of the proposed constitutive equation was evaluated against the experimental observations over the entire testing range.

\section{Material and experimental procedure}

\subsection{Material}

The chemical compositions of the selected PS and LCS are listed in Table 1. The PS/LCS bimetal was produced by centrifugal composite casting technology with the PS as the inner layer and the LCS as the outer layer. Since the different physical properties of the components, pouring temperature of the steels and volume ratio of PS to LCS must be carefully chosen to minimise the casting defects and achieve sound metallurgical bonding. Xiong et al. [23] produced the bimetal with high chromium cast iron (HCCI) and medium carbon steel (MCS) by sand mould casting technology. The results showed that there is an ideal volume ratio of HCCI to MCS for realising metallurgical bonding and preventing less or over bonding. For the current studied bimetal, the volume ratio of PS to LCS was determined as 2.55, which led to the thickness of PS and LCS layers are $\sim 18$ and $\sim 6 \mathrm{~mm}$, respectively. The detailed information about the composite casting procedure has been reported in elsewhere [5]. Fig. 1 shows the photographs and microstructure characteristics of the cast bimetallic billet. It is interesting to note that four different etched features were revealed in the cross section across the interface (Fig. 1(b)), in which the etched sub-layers showing in the PS layer were totally unexpected. As shown in Fig. 1(e) 
and (f), these sub-layers are fresh fully pearlitic (FP) region and conventional pro-eutectoid ferritic/pearlitic (pro-F/P) region, respectively. According to the Fe-C equilibrium phase diagram, the fully pearlitic microstructure usually cannot be obtained in hypo eutectoid steels unless a special treatment $[24,25]$ is applied. The reason for the formation of this microstructure in the studied bimetal has been discussed in our previous work [5]. In Fig. 1(c), the LCS layer is characterised by coarse ferrite and pearlitic patches. Additionally, the interface between the components is clearly displayed by the differences in contrast and microstructural features (Fig. 1(b) and (d)). There is no defect such as void, hole and less or over bonding was detected along the interface, which means the sound metallurgical bonding was achieved under the specified volume ratio. Fig. 2 shows the microhardness variation of the bimetal by averaging 10 measurements in different microstructural regions, respectively. The prominent hardness gradient between the LCS and PS layers indicates that the unique microstructural distribution, which arouses the microhardness variation, probably results in discontinuity of stress distribution during the hot deformation. So investigation on the constitutive flow behaviour of the bimetal is highly recommended.

\subsection{Isothermal compression test procedure}

The high temperature flow behaviour of the PS/LCS bimetal was investigated by means of hot compression tests. Cylindrical specimens with $20 \mathrm{~mm}$ in length and $10 \mathrm{~mm}$ in diameter were prepared from the same bimetallic billet. The thickness of the LCS and PS layers are $\sim 5$ and $\sim 15 \mathrm{~mm}$, respectively. The interface region, as shown in Fig. 1(b), is perpendicular to the axis. A series of compression tests were performed on a Gleeble 3500 thermo mechanical simulator, which is a fully integrated digital closed loop control thermal and mechanical testing system [26]. The test temperature range is $800-1100{ }^{\circ} \mathrm{C}$ with an interval of $100{ }^{\circ} \mathrm{C}$ while the equivalent strain rates are $0.02,0.1,1$ and 10 $\mathrm{s}^{-1}$. Prior to testing, a tantalum foil combined with a graphite foil was placed between the anvil and the specimen to reduce the friction. The specimens were heated to the deformation temperature at $20{ }^{\circ} \mathrm{C} / \mathrm{s}$ and held for $60 \mathrm{~s}$ to eliminate the thermal gradients without increasing element diffusion across the interface. Afterwards, $60 \%$ of overall reduction was conducted. During the hot compression, the 
microprocessor of Gleeble 3500 collected the load-stroke data automatically and converted to the true stress-strain curves using standard equations.

\section{Results and discussion}

\subsection{Flow behaviour of the PS/LCS bimetal}

Fig. 3 depicts the true stress-strain curves for the PS/LCS bimetal deformed at various strain rates and temperatures. In general, the flow behaviour was characterised by an increase of the true stress with true strain up to a peak or multi peaks that was followed by flow softening until the end of hot deformation. This typical profile shows the dynamic competition between the work-hardening caused by dislocation multiplication and interaction and the softening caused by dynamic recovery (DRV) or dynamic recrystallisation (DRX) $[6,27]$. However, it is difficult to define which component predominates the hot deformation because of different thresholds for DRV and DRX of the PS and LCS. In addition, the flow stress level at a certain plastic strain increases with an increase of strain rates or decrease of temperatures, which manifests the hot deformation is thermally activated $[7,13]$. When the critical strain rate and temperature were reached, DRX may dominate the flow behaviour rather than work-hardening or DRV. Accordingly, three types of true stress-strain curves that represent the dissipative nature are observed in Fig. 3: (i) a plateau type as shown in Fig. 3(a) and (b), where DRV may run through the softening process at low temperatures and high strain rates, (ii) single peak type at low temperature/low strain rate (Fig. 3(a) and (b)) and high temperature/high strain rate (Fig. 3(c) and (d)), which presents a steady state or continuous softening after peak because of continuous DRX [28, 29], (iii) multi peaks type at high temperatures and low strain rates as shown in Fig. 3(c) and (d) indicates the discontinuous DRX $[28,29]$.

Fig. 4 shows the microstructures of the interface region when the specimens were deformed at 900 ${ }^{\circ} \mathrm{C} / 10 \mathrm{~s}^{-1}, 900{ }^{\circ} \mathrm{C} / 0.1 \mathrm{~s}^{-1}, 1100{ }^{\circ} \mathrm{C} / 10 \mathrm{~s}^{-1}$ and $1100{ }^{\circ} \mathrm{C} / 0.1 \mathrm{~s}^{-1}$, respectively. Deformation at low temperature and high strain rate, as shown in Fig. 4(a), increased dislocation density and produced fine grain size. This microstructure contributes to high level of flow stress. The restoration mechanism of 
DRX was further activated by increasing temperature and decreasing strain rate. Grain growth is clearly observed in Fig. 4(b), (c) and (d).

Fig. 5 shows the typical appearance of the specimens deformed at $0.1 \mathrm{~s}^{-1}$ with temperatures of 800,900 , 1000 and $1100{ }^{\circ} \mathrm{C}$. Significant macro-heterogeneity of plastic deformation was observed when the temperature is $800{ }^{\circ} \mathrm{C}$. The extrusion of LCS during compression indicates the isostress behaviour of the bimetal. Although this phenomenon is usually approached for thick layer systems [30], transition from isostress behaviour to quasi isostrain behaviour with slight barrelling was occurred when the temperature increased to 900,1000 and $1100{ }^{\circ} \mathrm{C}$. Similar results were found at other strain rates, which means the extrusion of LCS from the studied bimetal is temperature sensitive and strain rate insensitive. Consequently, in order to deform the PS/LCS bimetal like a monolithic material, the appropriate processing temperature should be higher than $800{ }^{\circ} \mathrm{C}$, so that the isostrain flow behaviour dominates the hot deformation.

\subsection{Constitutive analysis of the flow behaviour}

Based on the analysis of the experimental flow behaviour of the bimetal, it was noted that the flow stress significantly depends on temperature $(T)$, strain rate $(\varepsilon)$ and plastic strain $(\varepsilon)$. Hence the proposed constitutive equation should be considerate and correctly describe the natural relationship between the parameters. Although the isostress behaviour occurred at $800{ }^{\circ} \mathrm{C}$, the prediction was conducted based on the initial assumption that an isostrain condition exited because of the isostrain behaviour prevalent at 900,1000 and $1100{ }^{\circ} \mathrm{C}$. As is well established, the correlation between the flow stress $(\sigma), T$ and $\dot{\varepsilon}$ at a given $\varepsilon$ can be expressed by an Arrhenius-type equation [21, 31] as Eq. (1), while the combined effects of $T$ and $\dot{\varepsilon}$ on the hot deformation behaviour can be represented by the Zener-Hollomon parameter (Z) [32] as formulated in Eq. (2).

$$
\dot{\varepsilon}=A f(\sigma) \exp \left(-\frac{Q}{R T}\right)
$$




$$
Z=\operatorname{\varepsilon exp}\left(\frac{Q}{R T}\right)
$$

where

$$
f(\sigma)=\left\{\begin{array}{llr}
\sigma^{n^{\prime}} & \alpha \sigma<0.8 & \text { (power law) } \\
\exp (\beta \sigma) & \alpha \sigma>1.2 & \text { (exponential law) } \\
{[\sinh (\alpha \sigma)]^{n}} & \text { for all } \sigma & \text { (hyperbolic law) }
\end{array}\right.
$$

In Eqs. (1), (2) and (3), $A$ is the material constant, $f(\sigma)$ is the flow stress function, $Q$ is the deformation activation energy $\left(\mathrm{J} \mathrm{mol}^{-1}\right), R$ is the universal gas constant $\left(8.314 \mathrm{~J} \mathrm{~mol}^{-1} \mathrm{~K}^{-1}\right), T$ is the absolute temperature (K), $\alpha$ and $\beta$ are the stress multipliers, $n$ and $n$ ' are the stress exponents. In Eq. (3), $\alpha=\beta / n^{\prime}$.

Substituting Eq. (1) and the hyperbolic law of $f(\sigma)$ into Eq. (2), the relationship between $Z$ and $\sigma$ is obtained as follows,

$$
Z=A[\sinh (\alpha \sigma)]^{n}
$$

Taking the logarithm of both sides of Eq. (4) and employing the definition of the hyperbolic law, the flow stress at a certain plastic strain can be written as a function of Zener-Hollomon parameter,

$$
\begin{aligned}
& \ln Z=\ln A+n \ln [\sinh (\alpha \sigma)] \\
& \sigma=\frac{1}{\alpha} \ln \left\{\left(\frac{Z}{A}\right)^{1 / n}+\left[\left(\frac{Z}{A}\right)^{2 / n}+1\right]^{1 / 2}\right\}
\end{aligned}
$$

In order to solve Eq. (6), the experimental stress-strain data were used to determine the material parameters of $\alpha, n, Q$ and $A$. The following is taking the true strain of 0.4 as an example to introduce the solution procedures for determination of the material parameters.

For the low and high stress levels, substituting the power law and the exponential law of $f(\sigma)$ into Eq.

(1) gives the following relationships, respectively.

$$
\begin{array}{ll}
\dot{\varepsilon}=A^{\prime} \sigma^{n^{\prime}} & \alpha \sigma<0.8 \\
\dot{\varepsilon}=A^{\prime \prime} \exp (\beta \sigma) & \alpha \sigma>1.2
\end{array}
$$


where, $A$ ' and $A$ ', are the material constants at a given deformation temperature. Taking the logarithm of the two sides of Eqs. (7) and (8), respectively, yields,

$$
\begin{aligned}
& \ln \dot{\varepsilon}=\ln A^{\prime}+n^{\prime} \ln \sigma \\
& \ln \dot{\varepsilon}=\ln A^{n}+\beta \sigma
\end{aligned}
$$

Then, substituting the values of strain rate and corresponding experimental flow stress at the true strain of 0.4 into Eqs. (9) and (10), the relationships of $\ln \dot{\varepsilon}-\ln \sigma$ and $\ln \dot{\varepsilon}-\sigma$ at different temperatures can be presented in Fig. 6(a) and (b), respectively. It is obvious that a well linear dependence between the flow stress and strain rate was revealed by the well-fitting lines, and the fitting lines are almost parallel at different deformation temperatures. This parallel indicates the relationships of $\ln \dot{\varepsilon}-\ln \sigma$ and $\ln \dot{\varepsilon}-$ $\sigma$ are temperature independent. By averaging the slops of the fitting lines in Fig. 6(a) and (b), the mean values of $n$ ' and $\beta$ were computed as 7.1439 and $0.0567 \mathrm{MPa}^{-1}$, respectively. Thus the value of $\alpha=\beta / n^{\prime}=0.0079 \mathrm{MPa}^{-1}$.

For all stress levels, substituting the hyperbolic law of $f(\sigma)$ into Eq. (1) gives,

$$
\dot{\varepsilon}=A[\sinh (\alpha \sigma)]^{n} \exp \left(-\frac{Q}{R T}\right)
$$

Taking the logarithm of the two sides of Eq. (11) and partial differentiating by temperature and strain rate leads to,

$$
n=\left[\frac{\partial \ln \dot{\varepsilon}}{\partial \ln [\sinh (\alpha \sigma)]}\right]_{T}
$$

and

$$
Q=R n\left[\frac{\partial \ln [\sinh (\alpha \sigma)]}{\partial(1 / T)}\right]_{\tilde{\xi}}
$$

Then, substituting the strain rate and true stress values (when $\varepsilon=0.4$ ) into Eq. (12), the relationship between $\ln \dot{\varepsilon}$ and $\ln [\sinh (\alpha \sigma)]$ at different temperatures was plotted in Fig. 7(a). By averaging the slops of the parallel fitting lines, the mean value of $n$ was calculated as 5.1923. In the same way, the relationship between $\ln [\sinh (\alpha \sigma)]$ and temperature at different strain rates, as shown in Fig. 7(b), can 
be obtained by substituting the true stress and temperature values into Eq. (13). Thereby the mean value of $Q$ was evaluated as $270.381 \mathrm{KJ} \mathrm{mol}^{-1}$ when the plastic strain reached 0.4 .

Using the solutions of $\alpha$ and $Q$, the relationship between $\ln Z$ and $\ln [\sinh (\alpha \sigma)]$ as formulated in Eq. (5) can be plotted in Fig. 8(a) at the true strain of 0.4. The good linear fit indicates the value of $\ln A$ is the intercept of the fitting line, which was found to be 25.2436 and $A$ value was $9.19 \mathrm{E}+10 \mathrm{~s}^{-1}$. Fig. 8(b) shows the variation of $Z$ value at different temperatures and strain rates at the given plastic strain of 0.4. It is evident that the $Z$ parameter decreases with increasing temperature and decreasing strain rate. This variation is similar to that of the flow stress as shown in Fig. 3. In addition, previous research [33] has demonstrated that the DRX softening mechanism is generally favoured at low $Z$ parameter, which means the high deformation temperatures and low strain rates are preferred in the current study. The observation in Fig. 4 is in line with Fig. 8(b).

Repeating the above solving procedures under different deformation strains, the compensation of the strain on the prediction of the flow stress can be incorporated into Eq. (6) by introducing the material parameter functions of $\alpha(\varepsilon), n(\varepsilon), Q(\varepsilon)$ and $A(\varepsilon)$. Fig. 9 shows the values of $\alpha, n, Q$ and $A$ calculated at different true stains within the range of $0.05-0.80$ in the interval of 0.05 . The polynomial fitting method was employed to fit these values. By varying the order of the polynomial from 2 to 9 , it was found that a sixth order polynomial may well represent the influence of true strain on the material parameters with a very good correlation $\left(\mathrm{R}^{2}>0.99\right)$ and generalisation. The fitting results are provided in Eq. (14) and Table 2.

$$
\left\{\begin{array}{l}
\alpha(\varepsilon)=B_{0}+B_{1} \varepsilon+B_{2} \varepsilon^{2}+B_{3} \varepsilon^{3}+B_{4} \varepsilon^{4}+B_{5} \varepsilon^{5}+B_{6} \varepsilon^{6} \\
n(\varepsilon)=C_{0}+C_{1} \varepsilon+B C_{2} \varepsilon^{2}+C_{3} \varepsilon^{3}+C_{4} \varepsilon^{4}+C_{5} \varepsilon^{5}+C_{6} \varepsilon^{6} \\
Q(\varepsilon)=D_{0}+D_{1} \varepsilon+D_{2} \varepsilon^{2}+D_{3} \varepsilon^{3}+D_{4} \varepsilon^{4}+D_{5} \varepsilon^{5}+D \varepsilon^{6} \\
A(\varepsilon)=L_{0}+L_{1} \varepsilon+L_{2} \varepsilon^{2}+L_{3} \varepsilon^{3}+L_{4} \varepsilon^{4}+L_{5} \varepsilon^{5}+L_{6} \varepsilon^{6}
\end{array}\right.
$$

Therefore, the constitutive equation for hot deformation behaviour of the PS/LCS bimetal with consideration of temperature, strain rate and plastic strain was summarised as follows, 


$$
\sigma(T, \dot{\varepsilon}, \varepsilon)=\frac{1}{\alpha(\varepsilon)} \ln \left\{\left(\frac{Z[T, \dot{\varepsilon}, Q(\varepsilon)]}{A(\varepsilon)}\right)^{1 / n(\varepsilon)}+\left[\left(\frac{Z[T, \dot{\varepsilon}, Q(\varepsilon)]}{A(\varepsilon)}\right)^{2 / n(\varepsilon)}+1\right]^{1 / 2}\right\}
$$

3.3. Verification of the proposed constitutive equation

In order to verify the accuracy of the proposed constitutive equation, predicted values of the flow stress over the entire temperature and strain rate ranges within the true strain of $0.05-0.8$ by an interval of 0.05 were calculated using Eq. (15). Thereafter, a comparison between the experimental and calculated results, as shown in Fig. 10, was carried out. A satisfactory match was confirmed in most of the cases except that the acceptable deviations were observed at $800{ }^{\circ} \mathrm{C} / 1 \mathrm{~s}^{-1}, 900{ }^{\circ} \mathrm{C} / 10 \mathrm{~s}^{-1}$ and $1000{ }^{\circ} \mathrm{C} / 0.02 \mathrm{~s}^{-1}$. These deviations may be attributed to the accumulated errors during the mathematical processes and the scatter of the experimental measurements. In addition, Fig. 10(a) shows that even though the isostress behaviour occurred at $800{ }^{\circ} \mathrm{C}$, the proposed equation based on the isostrain condition is still valid to reproduce the flow pattern at this temperature.

Further verifications were proceeded in terms of standard statistical parameters including correlation coefficient (R) and average absolute relative error (AARE), which are expressed as following equations,

$$
\begin{gathered}
\mathrm{R}=\frac{\sum_{i=1}^{i=N}\left(\sigma_{e}^{i}-\bar{\sigma}_{e}\right)\left(\sigma_{c}^{i}-\bar{\sigma}_{c}\right)}{\sqrt{\sum_{i=1}^{i=N}\left(\sigma_{e}^{i}-\bar{\sigma}_{e}\right)^{2} \sum_{i=1}^{i=N}\left(\sigma_{c}^{i}-\bar{\sigma}_{c}\right)^{2}}} \\
A A R E=\frac{1}{N} \sum_{i=1}^{i=N}\left|\frac{\sigma_{e}^{i}-\sigma_{c}^{i}}{\sigma_{e}^{i}}\right| \times 100
\end{gathered}
$$

where $N$ is the total number of data used in this study, $\sigma_{e}$ is the experimental flow stress, $\sigma_{c}$ is the calculated flow stress, $\bar{\sigma}_{e}$ and $\bar{\sigma}_{c}$ are the mean values of the $\sigma_{e}$ and $\sigma_{c}$, respectively.

Although the correlation coefficient is a common parameter used to measure the strength of linear relationship between the experimental and predicted results, the higher value of $\mathrm{R}$ may not always 
imply a better performance of the equation or model because of the biased tendency towards higher or lower values [34, 35]. So the average absolute relative error, which is an unbiased statistical parameter, was also computed through a term by term comparison of the relative error. Fig. 11 shows a good correlation between the experimental and calculated flow stresses with the $\mathrm{R}$ value as high as 0.9873 . Even though there are some relative larger deviations occurred in range of 160-220 MPa, the value of $A A R E$ was found to be only $4.81 \%$. High value of $\mathrm{R}$ combined with low value of $A A R E$ indicates that an excellent predictability of the equation or model. Therefore, it can be concluded that the proposed constitutive equation gives an accurate and reliable estimate for the hot deformation behaviour of the studied PS/LCS bimetal.

\section{Conclusions}

Hot deformation behaviour of the novel PS/LCS bimetal in a wide range of temperatures $\left(800-1100{ }^{\circ} \mathrm{C}\right)$, strain rates $\left(0.02-10 \mathrm{~s}^{-1}\right)$ and strains $(0.05-0.8)$ has been investigated via physical experiment and constitutive model. The following conclusions are drawn from this work.

(1) The unique layered microstructure characteristics and significant hardness gradient between the layers make a lot of sense to study the high temperature flow behaviour of the novel PS/LCS bimetal.

(2) The true stress-strain curves revealed that the flow stress of the bimetal is substantially sensitive to temperature and strain rate as well as plastic strain. Three types of curve patterns displayed their influences by the typical profile of dynamic competition between work-hardening and DRV/DRX, which was demonstrated by microstructural investigations.

(3) Based on the experimental results, a constitutive model was developed using the phenomenological method in which the effects of temperature and strain rate were considered in Zener-Hollomon parameter while the compensation of plastic strain was incorporated in material parameters of $\alpha, n, Q$ and $A$.

(4) The proposed constitutive equation can accurately reproduce the hot deformation behaviour of the PS/LCS bimetal. High value of correlation coefficient $(\mathrm{R}=0.9873)$ combined with low value of average absolute relative error $(A A R E=4.81 \%)$ suggests that the constitutive model is valid and reliable. 


\section{Acknowledgements}

The authors appreciate Mr. Bob de Jong and Dr. Liang Chen for their kind assistance and help during hot compression tests. The first author also wishes to thank the China Scholarship Council (CSC) for supporting the current research work.

\section{References}

[1] B. Strnadel, P. Hausild, Mat. Sci. Eng. A 486 (2008) 208-214.

[2] C.M. Bae, W.J. Nam, C.S. Lee, Scripta. Mater. 41 (1999) 605-610.

[3] H. Sakamoto, K. Toyama, K. Hirakawa, Mat. Sci. Eng. A 285 (2000) 288-292.

[4] Y.D. Chung, H. Fujii, R. Ueji, N. Tsuji, Scripta. Mater. 63 (2010) 223-226.

[5] X.J. Gao, Z.Y. Jiang, D.B. Wei, H.J. Li, S.H. Jiao, J.T. Han, in: The Fifth Baosteel Biannual Academic Conference, Shanghai, China, 2013, pp. C110-116.

[6] Y.C. Lin, M.S. Chen, J. Zhong, Comput. Mater. Sci. 42 (2008) 470-477.

[7] Y.C. Lin, G. Liu, Comput. Mater. Sci. 48 (2010) 54-58.

[8] Y.C. Lin, Y.C. Xia, X.M. Chen, M.S. Chen, Comput. Mater. Sci. 50 (2010) 227-233.

[9] S. Mandal, V. Rakesh, P.V. Sivaprasad, S. Venugopal, K.V. Kasiviswanathan, Mat. Sci. Eng. A 500 (2009) 114-121.

[10] D. Samantaray, S. Mandal, A.K. Bhaduri, Mater. Design. 31 (2010) 981-984.

[11] H.Y. Wu, J.C. Yang, F.J. Zhu, H.C. Liu, Mat. Sci. Eng. A 550 (2012) 273-278.

[12] H.Y. Wu, J.C. Yang, F.J. Zhu, C.T. Wu, Mat. Sci. Eng. A 574 (2013) 17-24.

[13] A. He, L. Chen, C. Wang, L. Huangfu, Mater. Design. 46 (2013) 54-60.

[14] C.H.J. Davies, E.B. Hawbolt, I.V. Samarasekera, J.K. Brimacombe, J. Mater. Process. Tech. 70 (1997) 244-251.

[15] S. Spigarelli, E. Evangelista, E. Cerri, T.G. Langdon, Mat. Sci. Eng. A 319-321 (2001) 721-725.

[16] J.C. Shao, B.L. Xiao, Q.Z. Wang, Z.Y. Ma, Y. Liu, K. Yang, Mat. Sci. Eng. A 527 (2010) 7865-7872.

[17] Z.J. Wang, L.H. Qi, J.M. Zhou, J.T. Guan, J. Liu, Comput. Mater. Sci. 50 (2011) 2422-2426.

[18] M.R. Rokni, A. Zarei-Hanzaki, A.A. Roostaei, A. Abolhasani, Mater. Design. 32 (2011) 4955-4960.

[19] F. Yin, L. Hua, H.J. Mao, X.H. Han, Mater. Design. 43 (2013) 393-401.

[20] J.Q. Zhang, H.S. Di, X.Y. Wang, Y. Cao, J.C. Zhang, T.J. Ma, Mater. Design. 44 (2013) 354-364.

[21] J.J. Jonas, C.M. Sellars, W.J.McG. Tagart, Int. Metal. Rev. 14 (1969) 1-24.

[22] F.A. Slooff, J. Zhou, J. Duszczyk, L. Katgerman, Scripta Mater. 57 (2007) 759-762.

[23] B. Xiong, C. Cai, B. Lu, J. Alloy. Compd. 509 (2011) 6700-6704.

[24] J.P. Houin, A. Simon, G. Beck, Trans. ISIJ. 21 (1981) 726-731.

[25] H.L. Yi, Mat. Sci. Eng. A 527 (2010) 7600-7604.

[26] Defining a new era in physical simulation and thermal-mechanical testing, http://gleeble.com.

[27] H.Z. Li, H.J. Wang, Z. Li, C.M. Liu, H.T. Liu, Mat. Sci. Eng. A 528 (2010) 154-160.

[28] A. Momeni, K. Dehghani, Mat. Sci. Eng., A 527 (2010) 5467-5473.

[29] M.J. Luton, C.M. Sellars, Acta Metall. 20 (1969) 1033-1043.

[30] D.R. Lesuer, C.K. Syn, O.D. Sherby, J. Wadsworth, J.J. Lewandowski, W. H. Hunt Jr, Int. Mater. Rev. 41 
(1996) 169-197.

[31] C.M. Sellars, W.J. McTegart, Acta Metallurgica 14 (1966) 1136-1138.

[32] C. Zener, H. Hollomon, J. Appl. Phys. 15 (1944) 22.

[33] B. Paul, R. Kapoor, J.K. Chakravartty, A.C. Bidaye, I.G. Sharma, A.K. Suri, Scripta. Mater. 60 (2009) 104-107.

[34] O. Sabokpa, A. Zarei-Hanzaki, H.R. Abedi, N. Haghdadi, Mater. Design. 39 (2012) 390-396.

[35] M.P. Phaniraj, A.K. Lahiri, J. Mater. Process. Tech. 141 (2003) 219-227. 


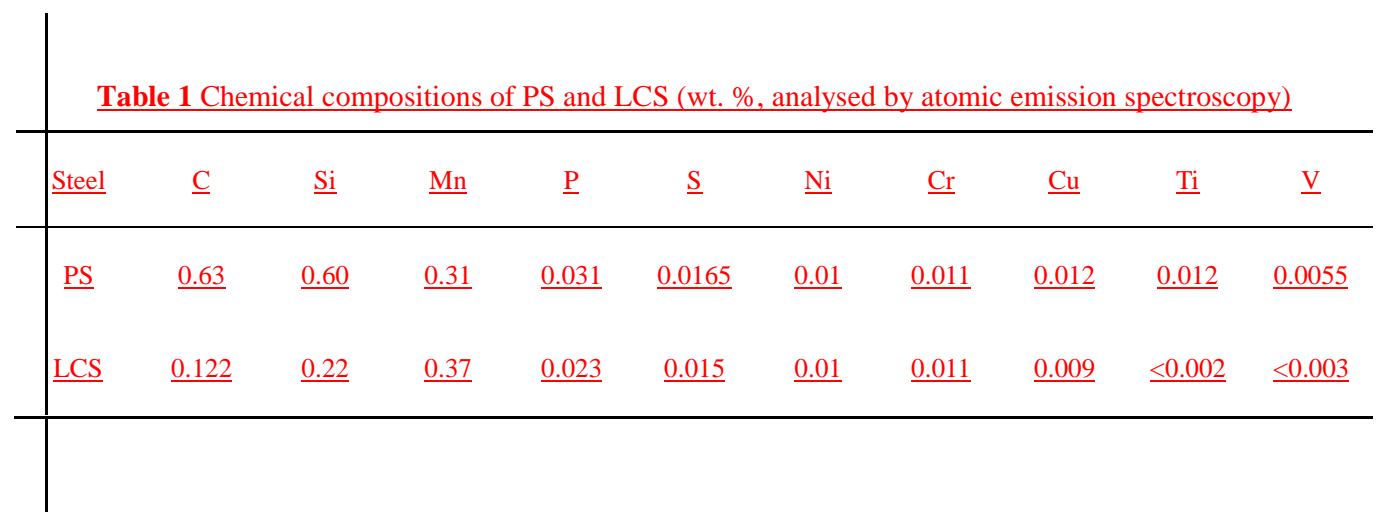


Table 2 Coefficients of the polynomial for $\alpha, n, Q$ and $A$.

\begin{tabular}{|c|c|c|c|c|c|c|c|}
\hline$\underline{\alpha}$ & & $\underline{n}$ & & $\underline{Q}$ & & $\underline{A}$ & \\
\hline$\underline{B}_{0}$ & $\underline{0.0154}$ & $\underline{C_{0}}$ & $\underline{6.8948}$ & $\underline{D}_{0}$ & $\underline{236.62}$ & $\underline{L_{0}}$ & $-6.522 E+10$ \\
\hline$\underline{B}_{1}$ & $\underline{-0.0966}$ & $\underline{C}_{1}$ & $\underline{0.0656}$ & $\underline{D}_{1}$ & $\underline{637.45}$ & $\underline{L_{1}}$ & $2.410 \mathrm{E}+12$ \\
\hline$\underline{B_{2}}$ & $\underline{0.5442}$ & $\underline{C_{2}}$ & $\underline{-78.606}$ & $\underline{D_{2}}$ & -4736.1 & $\underline{L_{2}}$ & $-1.718 \mathrm{E}+13$ \\
\hline$\underline{B}_{3}$ & $\underline{-1.6593}$ & $\underline{C_{3}}$ & $\underline{373.45}$ & $\underline{D}_{3}$ & $\underline{16514}$ & $\underline{L_{3}}$ & $\underline{5.754 \mathrm{E}+13}$ \\
\hline$\underline{B}_{4}$ & $\underline{2.8141}$ & $\underline{C}_{4}$ & -761.08 & $\underline{D}_{4}$ & $\underline{-29203}$ & $\underline{L}_{4}$ & $-9.517 \mathrm{E}+13$ \\
\hline$\underline{B}_{\underline{5}}$ & $\underline{-2.4639}$ & $\underline{C}_{5}$ & $\underline{731.57}$ & $\underline{D}_{5}$ & $\underline{25638}$ & $\underline{L_{5}}$ & $\underline{7.595 \mathrm{E}+13}$ \\
\hline$\underline{B}_{6}$ & $\underline{0.8646}$ & $\underline{C}_{6}$ & -269.76 & $\underline{D}_{6}$ & $\underline{-8861.4}$ & $\underline{L}_{6}$ & $-2.226 \mathrm{E}+13$ \\
\hline
\end{tabular}


(a)

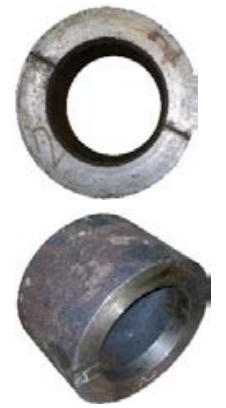

(b)

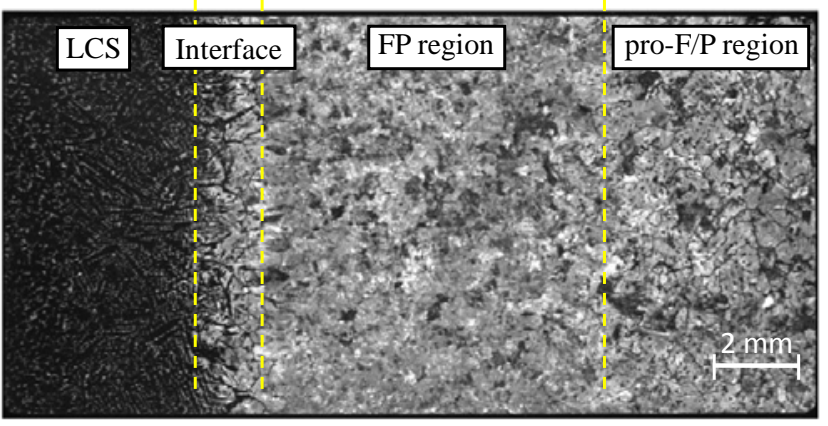

(c)

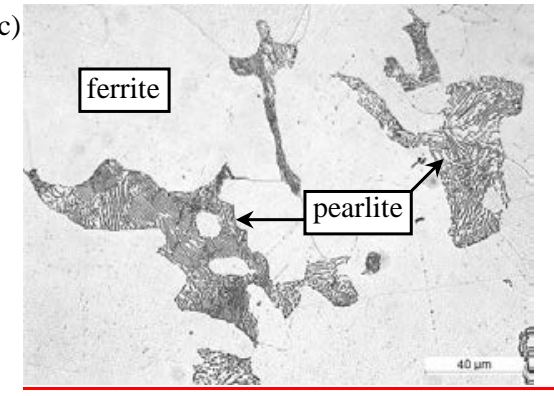

(e)

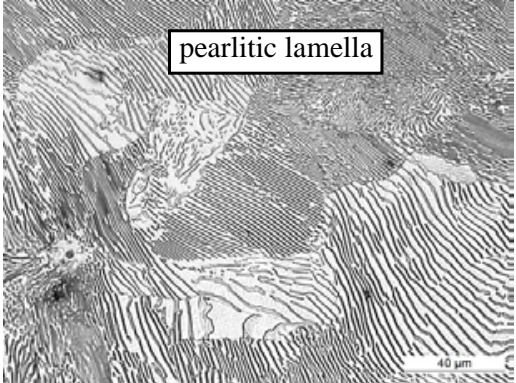

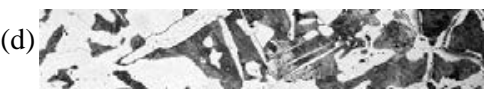
is sf ferrite $\rightarrow$ or 4 in

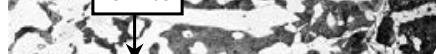

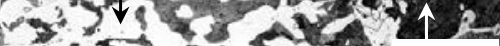
- 3 C pearlite

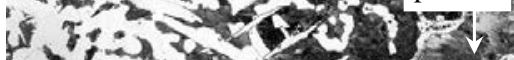

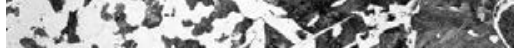

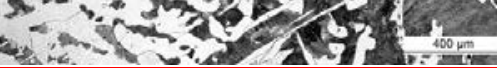

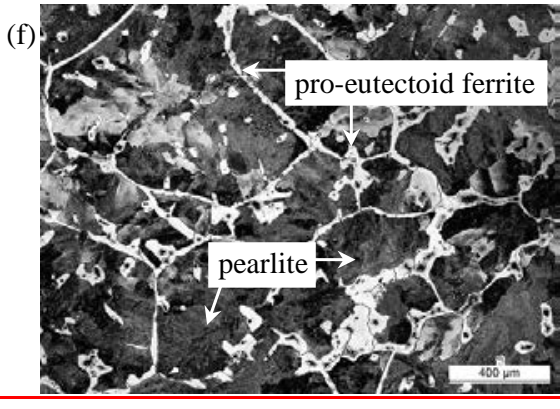

Fig. 1 Photographs and optical micrographs of the cast PS/LCS bimetal: (a) Photographs, (b) Overall cross section showing four different regions, (c) Microstructure of LCS layer, (d) Microstructure of interface, (e) Microstructure of FP region, (f) Microstructure of pro-F/P region. 


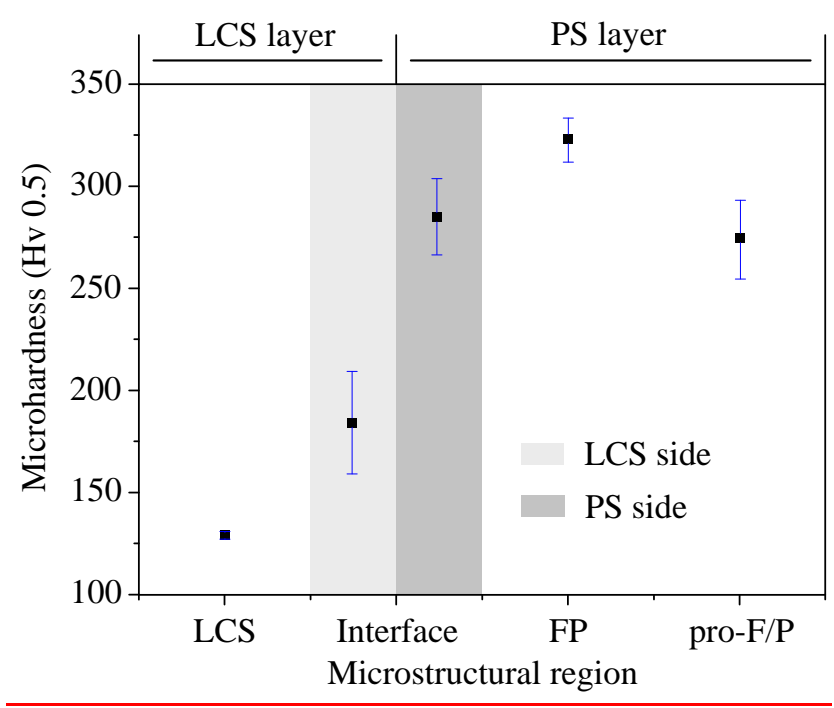

Fig. 2 Microhardness variation in the LCS and PS layers of the bimetal 

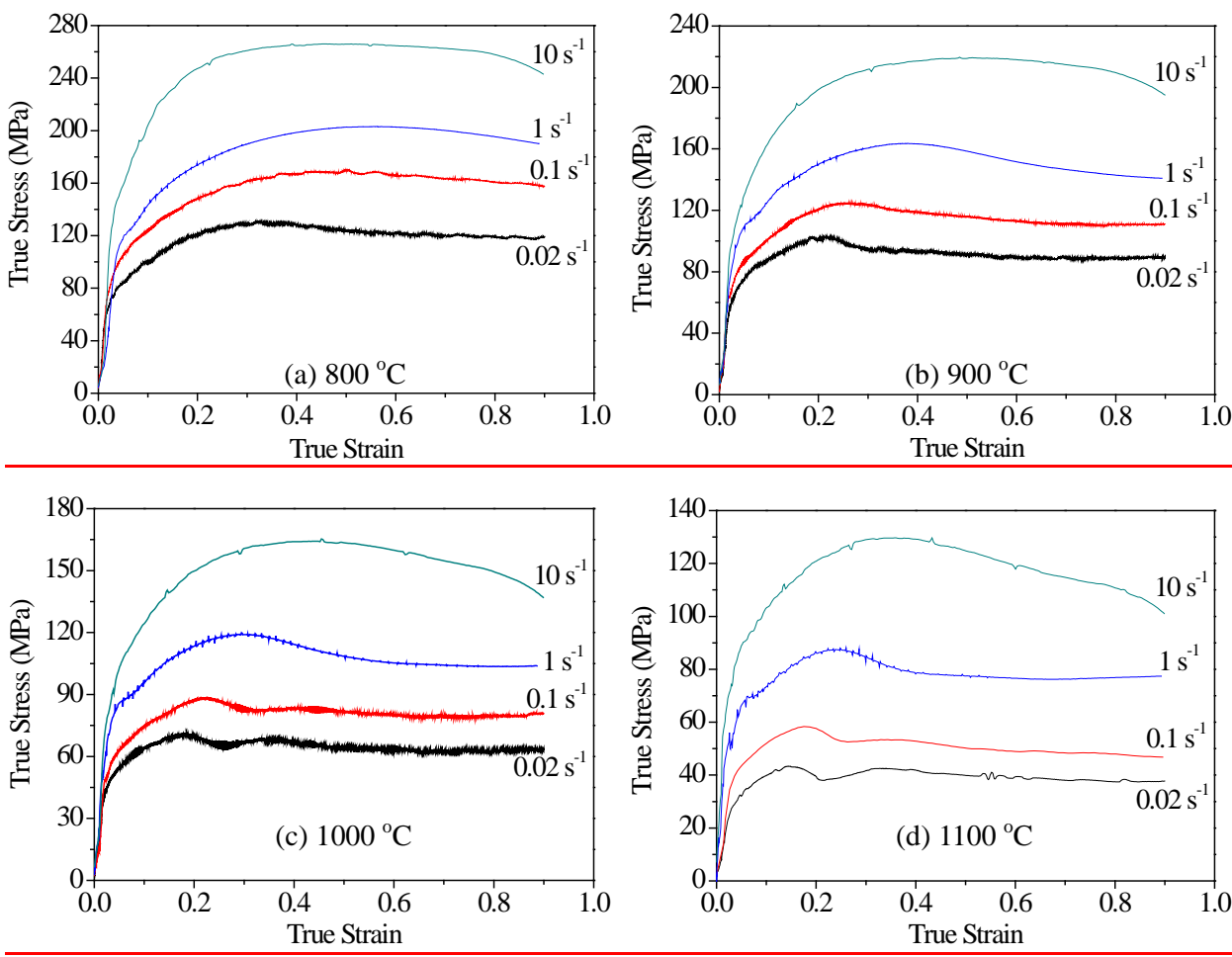

Fig. 3 True stress-strain curves of the PS/LCS bimetal subjected to different strain rates and

$\underline{\text { temperatures }}$ 

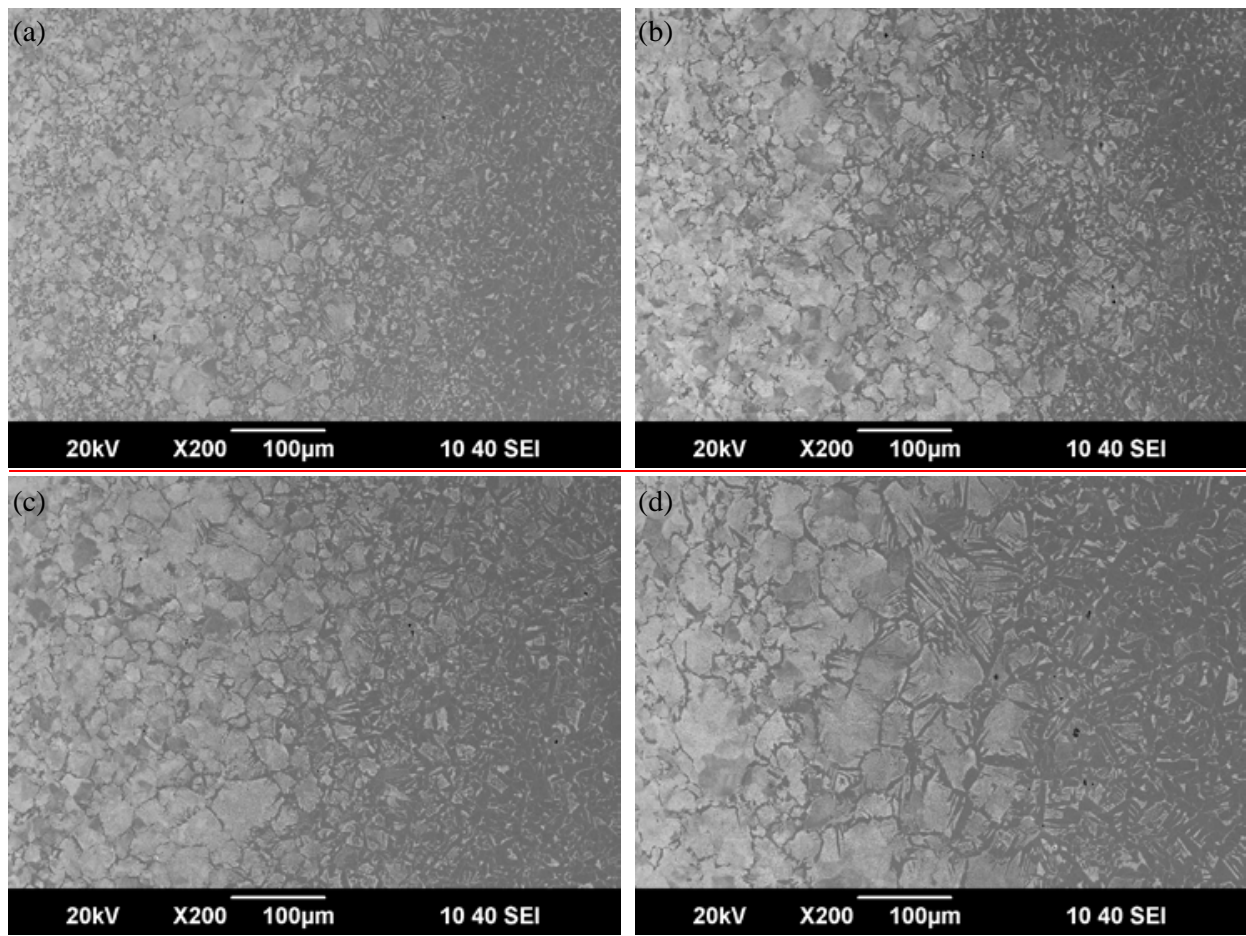

Fig. 4 SEM micrographs of the interface region after hot compression at: (a) $900{ }^{\circ} \mathrm{C} / 10 \mathrm{~s}^{-1}$, (b) 900 ${ }^{\circ} \mathrm{C} / 0.1 \mathrm{~s}^{-1}$, (c) $1100{ }^{\circ} \mathrm{C} / 10 \mathrm{~s}^{-1}$, (d) $1100{ }^{\circ} \mathrm{C} / 0.1 \mathrm{~s}^{-1}$. The left side is PS, and the right side is LCS; the gray phase is pearlite, and the dark phase is ferrite. 


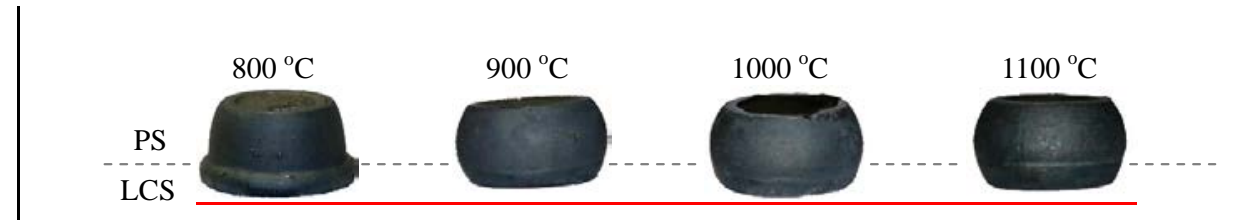

Fig. 5 Typical appearance of the specimens deformed at $0.1 \mathrm{~s}^{-1}$ with temperatures of 800, 900, 1000

and $1100^{\circ} \mathrm{C}$. Above the dash line is PS layer, and below the dash line is LCS layer. 

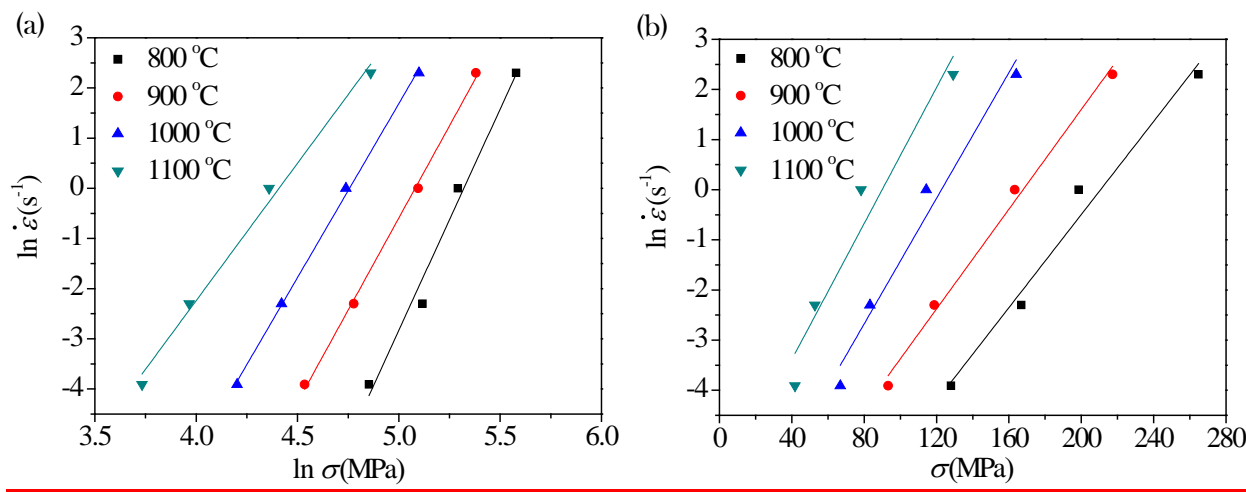

Fig. 6 Relationships between (a) $\ln \dot{\varepsilon}$ and $\ln \sigma$, (b) $\ln \dot{\varepsilon}$ and $\sigma$ when $\varepsilon=0.4$. The solid lines are linear fitting lines. 

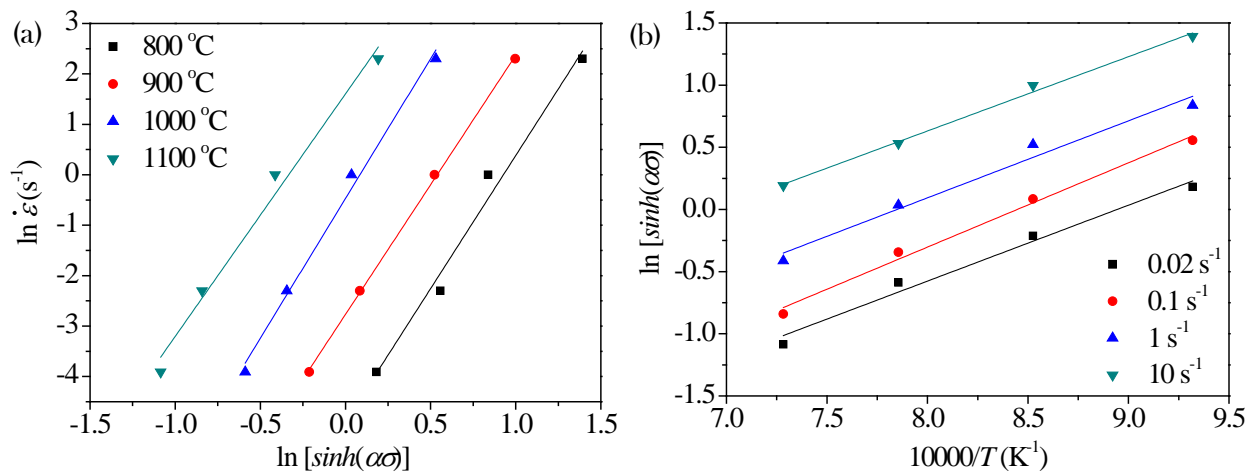

Fig. 7 Relationships between (a) $\ln \dot{\varepsilon}$ and $\ln [\sinh (\alpha \sigma)]$, (b) $\ln [\sinh (\alpha \sigma)]$ and temperature when $\varepsilon=0.4$.

The solid lines are linear fitting lines. 

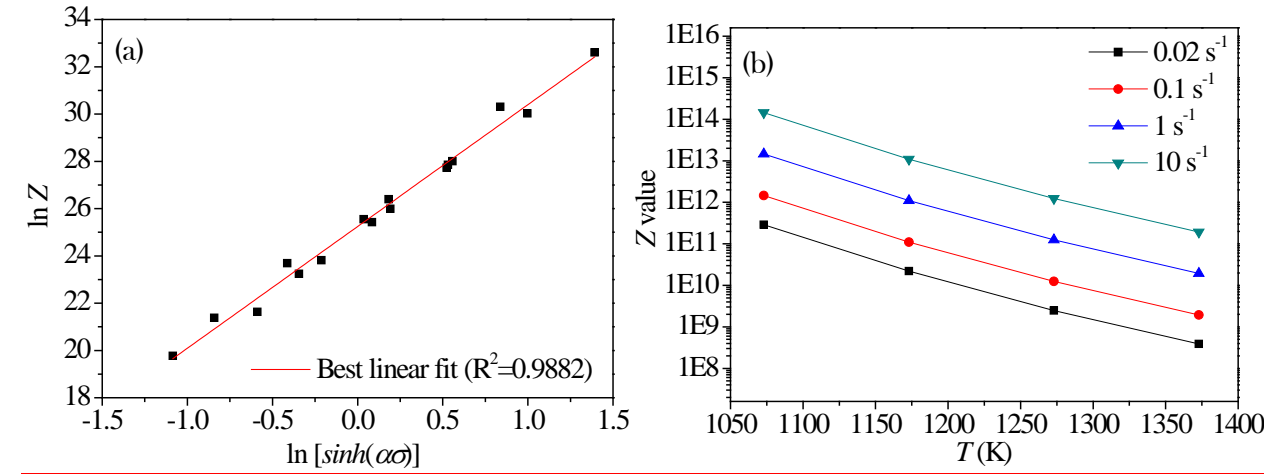

Fig. 8 Relationships between (a) $\ln Z$ and $\ln [\sinh (\alpha \sigma)]$, (b) $Z$ and temperature when $\varepsilon=0.4$. 

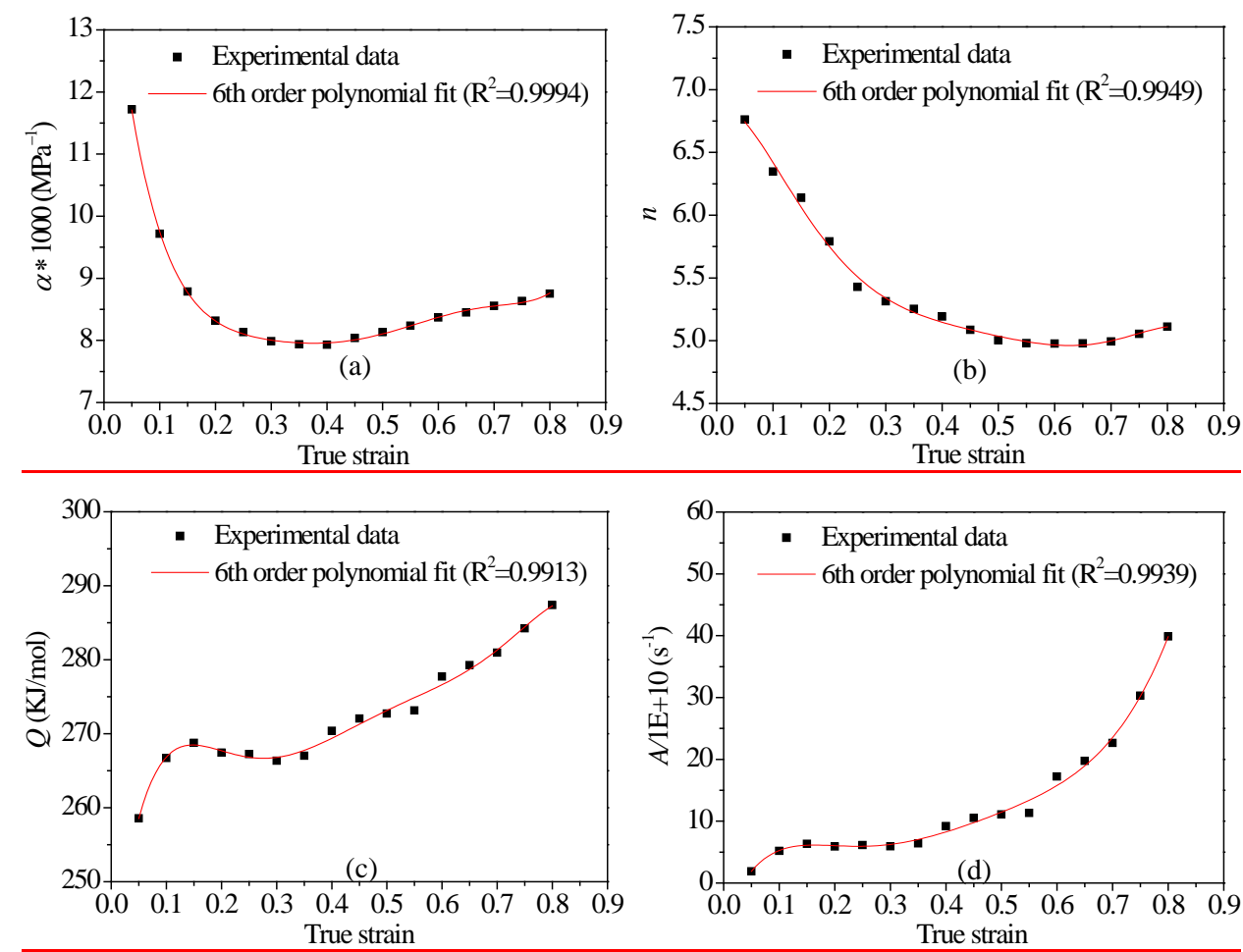

Fig. 9 Relationships between (a) $\alpha$, (b) $n$, (c) $Q$, (d) $A$ and true strain by polynomial fit of the PS/LCS

bimetal. 

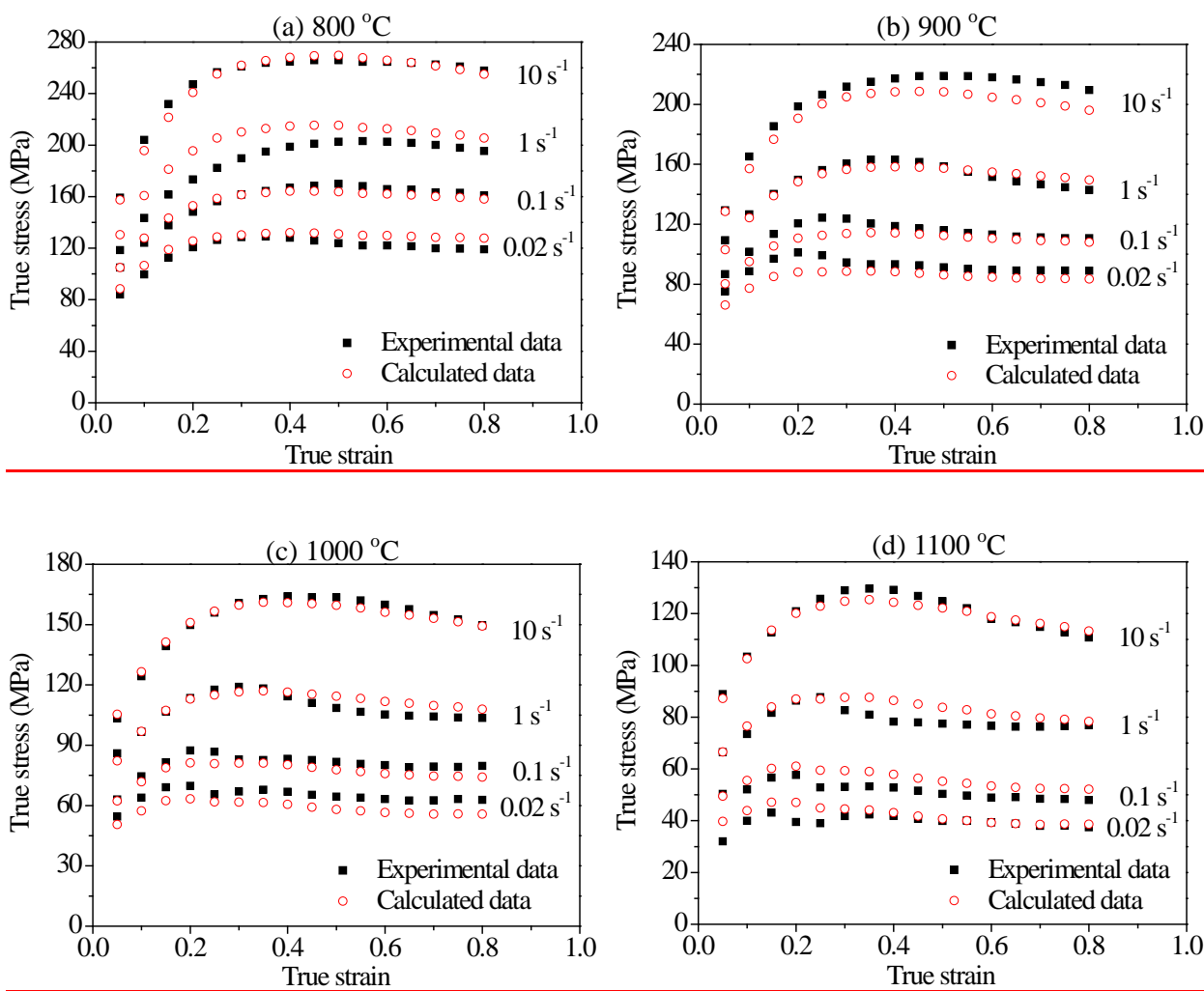

Fig. 10 Comparison between the calculated and experimental flow stress values of the PS/LCS bimetal

at different strain rates and temperatures 


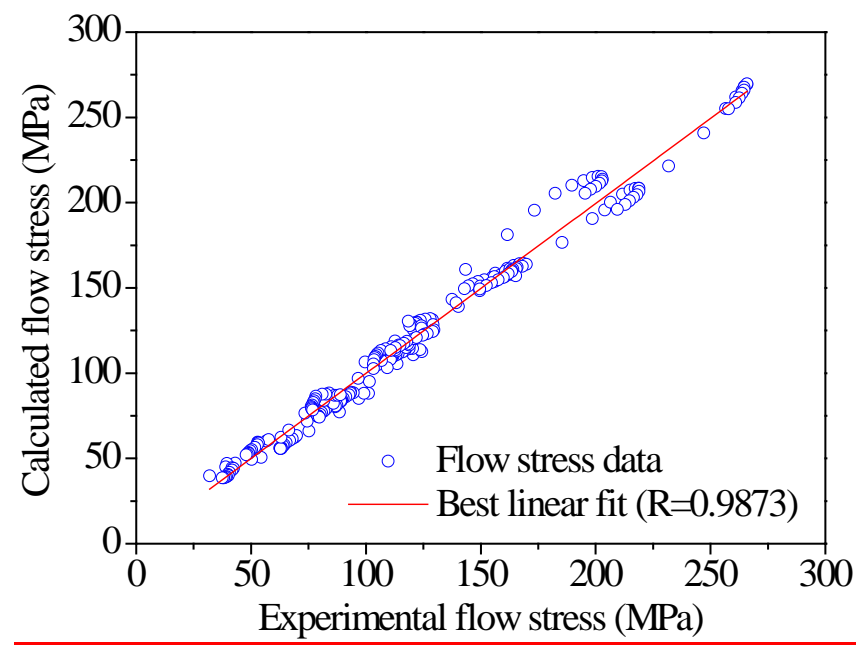

Fig. 11 Correlation between the experimental and calculated flow stress data from the proposed

constitutive equation

Formatted: English (Australia)

Formatted: Indent: Left: $0 \mathrm{~cm}$, First

line: $0 \mathrm{ch}$ 